\title{
Guided Imagery and Relaxation Audio for Children with Cancer: Development and Evaluation
}

\author{
Azizah Othman $^{1 *}$, Muhammad Junaidi Buang ${ }^{2}$, Mai Nurul Ashikin Taib ${ }^{1}$, \\ Norsarwany Mohamad ${ }^{1}$, Ariffin Nasir ${ }^{1}$ \\ ${ }^{1}$ Department of Pediatrics, Universiti Sains Malaysia, Kubang Kerian, Kelantan, Malaysia \\ ${ }^{2}$ ALIR PADU Enterprise, Kuala Selangor, Selangor, Malaysia \\ Email: *azeezah@kk.usm.my
}

Received March 21, 2013; revised April 20, 2013; accepted April 27, 2013

Copyright (c) 2013 Azizah Othman et al. This is an open access article distributed under the Creative Commons Attribution License, which permits unrestricted use, distribution, and reproduction in any medium, provided the original work is properly cited.

\begin{abstract}
The study aims to develop, validate and evaluate a Guided Imagery and Relaxation (GIR) audio in Bahasa Malaysia, serving as one of the coping tools for children with cancer. Based on extensive review, GIR script was developed by a clinical psychologist. Panels of experts including pediatric oncologists and psychiatrists conducted initial assessment at multiple stages of hearing sessions. The final version of the audio was pre-recorded in MP3 player for evaluation. Face and content validation of $3 \times$ approximately 5 minutes duration of GIR audio series were obtained via experts' reports. Twenty-three participants ( 5 adults, 18 children with cancer) listened to the audio and completed evaluation form. All adult evaluators gave positive remarks on the script, narration and recording quality. As for the children, $100 \%$ completed audio-hearing and majority $(66.7 \%-88.9 \%)$ were able to imagine well without falling asleep and in some way were positively affected by the imagery-induced relaxation audio. Majority was not disturbed during the hearing sessions (66.7\%), will hear it again (83.3\%) and found it enjoyable (88.8\%). This is the first known study developing GIR audio in Bahasa Malaysia which is feasible, enjoyable and beneficial for children that warrants efficacy study.
\end{abstract}

Keywords: Guided-Imagery Relaxation; Children; Cancer; Development

\section{Introduction}

Hospitalization is a highly distressing experience, particularly for children with chronic, debilitating illness such as cancer. Studies have continued to suggest that children with cancer have significantly more psychological problems than other children in the community [1]; they experience at different phases of the illness, due to multiple different factors. Psychological interventional tools, such as guided imagery and relaxation aid to improve coping with children of this population.

\section{Literature Review}

\subsection{Psychological Effects of Childhood Cancer}

There are multiple phases of the illness that may trigger emotional response in children whom are diagnosed to have cancer. Therapy for haematological malignancies consists of systemic chemotherapy which may span over 6 months to 3 years depending upon the stage of disease at diagnosis. Therapy is intensive in the first 2 months

\footnotetext{
${ }^{*}$ Corresponding author.
}

followed by intermediate phase and maintenance phase. Therapy for solid tumors consists of systemic chemotherapy with surgery and/or radiation therapy depending on the stage of disease at diagnosis. The therapy is intensive in the first 1 months followed by surgery and intermediate phase.

The initial phase is the most stressful [2] where the children are subjected to numerous tests before a definitive diagnosis is established. Later, learning that benign physical symptoms may actually be cancer represents a major crisis situation. Tolerating separation from family and friends and the pain and anxiety of invasive medical tests are major issues for the child [3].

Once treatment has been initiated, the child must make adjustment to complex treatment schedule, frequent hospital visits and repeated medical procedures, and at the same time tolerate drugs and treatments that are toxic and make people feel sick [2]. The multimodal approach to cancer treatment incorporates surgery, chemotherapy, and radiation therapy, alone or in combination to ensure the best therapeutic outcome and increase survival. Unfortunately, these treatments may be painful, disfiguring, 
frightening, and often have aversive side effects that can alter one's physical and emotional well-being. Nausea and vomiting being very common, has also been identified as one of the major cause of distress. Together with fatigue, pain and discomfort caused by the illness and its treatment can lead to a loss of interest in normal activities.

Other factors related to specific characteristics of the illness such as degree of severity, any restrictions or pain caused by the illness or uncertainty of the prognosis, and environmental and social factors, such as availability of appropriate schooling and responses of peers to the child may also affect the children's well-being negatively [4].

Psychological support is imperative to help children cope with the illness, and other stressful experience related to it. However, this need is often neglected due to limited time, resources, and necessary training on part of the healthcare providers. There are many psychological techniques promoting effective coping, and guided imagery and relaxation are one of those techniques.

\subsection{Guided Imagery Relaxation in Children}

Guided imagery refers to a range of techniques from simple visualization and direct imagery-based suggestion through metaphor and storytelling. It is a psychological intervention tool that generates positive sensation and emotion through mental imagination to induce a state of psychological and physiological relaxation. It is one form of self-regulation therapy during which a state of deep relaxation is induced using progressive muscle relaxation (PMR) which allows the subject to then be guided in actively creating images that facilitate resolution of certain targeted problems. This differs from hypnosis in that the child, through imagery, creates his own solution to the problem rather than the therapist offering suggestions for change. This process is felt to be especially effective in children because of their ability to have creative, active imaginations with a high degree of suggestibility [5-7].

Increasingly, child-development experts are recognizing the importance of imagination and the role it plays in understanding reality [8]. They proposed that the children learn and understand best when they are imagining, day-dreaming or role-playing. Imagination is necessary for learning about people and events we do not directly experience, such as history or events on the other side of the world. For young children, it allows them to ponder the future, such as what they want to do when they grow up.

\subsection{Clinical Evidence of Guided Imagery and Relaxation}

Guided imagery has been used increasingly by healthcare providers in the medical field with impressive results. This is particularly true not only with cancer patients but also with patients who have other medical concerns such as stroke, recurrent abdominal pain, osteoarthritis, asthma, and chronic fatigue syndrome [9]. The techniques have been shown to reduce anxiety in surgical patients. In cancer patients in particular, these psychological therapies have been proven to be effective in reducing patients' distress and widely accepted by both the patients and also physicians [10-13].

Interestingly, GIR has been identified as one of the ten most frequently recommended complementary cancer therapies [14]. Unfortunately, most of our paediatric population in general, and paediatric oncology patients in specific are not exposed to such psychologically directed therapy mostly due to unavailability of such a tool in local language.

\subsection{Objectives and Hypothesis}

The study's objective was to develop a GIR audio in Bahasa Malaysia as an interventional psychological tool for children and to pilot test it to paediatric oncology patients in hope to improve the patient's well-being as a whole. In particular, this paper focuses on the development and validation of the GIR audio as well as the evaluation of the acceptance of both paediatric oncology patients and child experts of various fields.

It was hypothesized that the Malay language guided imagery and relaxation audio is producible, acceptable to expert and participants, feasible and valid to be used as psychological intervention tool for Malaysian pediatric oncology children.

\section{Methods}

\subsection{Setting and Participants}

This is part of a larger study which was conducted at Health Campus, Hospital Universiti Sains Malaysia (HUSM). Two groups of participants involved, namely children with cancer and child experts. A total of 18 children participated in this study. They are children diagnosed with cancer and under active treatment for cancer. The inclusion criteria were the child is treated for any kind of cancer in HUSM, between 7 - 15 years old, is cognitively capable to participate in this study which means the child is generally able to understand instructions and attend school age appropriately, speaks and understand Bahasa Malaysia adequately, whom parent is consented, and commits to complete the entire audio hearing sessions. The exclusion criteria were the child is very sick during the study as verified by pediatric oncologists and whom parent is not consented. The dropped off criteria were child refuses to complete the audio hearing sessions, withdraws their consent, critically ill 
after enrollment and passed away before completion of study. The child experts include 2 pediatricians, 1 child psychiatrist, 1 parent and 1 staff nurse, chosen based on their substantial experience dealing with children with cancer.

\subsection{Development of Guided Imagery and Relaxation (GIR) in Audio in Bahasa Malaysia}

Based on extensive review [8,9,15] an imagery-induced relaxation script for children in Bahasa Malaysia was developed. The initial script consist of sequence of deep breathing instructions, followed by imagination involving multiple senses such as touch, smell, sight, sound and taste, and at the end there was component of healing before the child is brought back to reality. The whole script runs for about 30 minutes. Script, narration, and recording quality were assessed through hearing sessions. Hearing and evaluation sessions were conducted at multiple stages by panels of experts including psychologist, pediatric oncologists and child psychiatrist. Comments and suggestions for improvement were taken into consideration and changes were made accordingly. For example, a single script of 30 minutes running time was later divided into 3 parts, to capture adequate attention and concentration on part of the listener (i.e. child). Reconciliations were made and total of 4 times of audio recording had to be done before the final imagery-induced relaxation scripts were produced. Based on the script, an instrumental background music which was composed by a music teacher who has several years of experiences composing music for children was inserted. The script was then recorded into audio format and downloaded into MP3 player to be used as a tool in the main study.

The script was recited by a clinical psychologist with appropriate instrumental background music. The final version of GIR script started with breathing technique followed by a short progressive muscle relaxation sequence. Then the children were guided through a fantasy journey in which they imagine flying up high in the sky passing through the peaceful places. Incorporated in the script was the power of the Creator to promote the power of healing, feeling of reliant and subsequently perceived strength. Finally, they were brought back into the reality slowly by descending down from the sky. The GIR audio has 3 versions; each using different imagination stimuli namely "The Balloon”, “The Flower Garden” and "The Flying Carpet” but all consisted of similar setting and sequence. The runtime for each version is 5:35 minutes, 5:36 minutes and 5:26 minutes respectively. This relaxation technique was chosen because imaginative approach may induce relaxation, emotional comfort and somatic reactions indirectly by influencing children’s cognitions.

\subsection{Protocol and Procedures}

Approval from Universiti Sains Malaysia's ethical committee and written informed consent from the parents of the children were obtained. Children who fulfilled the inclusion criteria of the main study were recruited. A medical officer doing a postgraduate program in paediatrics and two psychology students were trained by the principal researcher who is a clinical psychologist to assist the child to operate the MP3 player to listen to the audio and to complete the evaluation questionnaire.

\subsection{Analysis}

Face and content validation of the GIR script was established through child experts' reports of the GIR audio as an intervention tool. A tool assessment form consisted of stem of items on appropriateness of the script, length and the sequencing, the narrator factors such as clarity of the tone of voice and pacing of the voice, and also the recording quality and background music. The experts were required to give comments on the GIR audio as a whole. In addition, both child experts and children with cancer completed the evaluation form after listening to the GIR audio.

\section{Results}

\subsection{Descriptive Analysis of Children Participants}

Eighteen $(\mathrm{N}=18)$ patients participated in this study. All patients were children diagnosed with various types of cancer and undergoing active treatment for cancer in the pediatric oncology ward 6 Utara, HUSM. No patients were excluded.

Majority of children were female (61.1\%) and with a mean age of 9 years old (range 6 - 13 years old). Out of 18 children, $61.1 \%$ are diagnosed to have leukemia and $50 \%$ of them are currently under intermediate phase of chemotherapy. The mean duration of illness for the children in this study was 9 months. As for the family background, most of them come from family with monthly income group in between Ringgit Malaysia (RM) RM1001.00 - RM5000.00 (44.4\%). Fifty percent of fathers working as laborer and majority of mothers were not working (55.6\%). The demographic data is presented in Table 1.

\subsection{Face and Content Validation}

Face and content validation were obtained via reports from experts. For this purpose, 2 pediatricians, 1 child psychiatrist, 1 parent, and 1 staff nurse were given MP3 prerecorded with GIR and were asked to give feedback 
Table 1. Demographic data of children participants.

\begin{tabular}{lll}
\hline Variables & & n (\%) \\
\hline Gender & Male & $7(38.9)$ \\
Type of cancer & Female & $11(61.1)$ \\
& Leukemia & $11(61.1)$ \\
& Lymphoma & $1(5.6)$ \\
Stage of chemotherapy & Solid tumor & $6(33.3)$ \\
& Induction & $1(5.6)$ \\
& Intermediate & $9(50.0)$ \\
Monthly income (RM) & Maintenance & $8(44.4)$ \\
& $<1000$ & $5(27.8)$ \\
Father's education level & 1001 - 5000 & $8(44.4)$ \\
& $>5000$ & $5(27.8)$ \\
Mother's education level & Secondary & $11(61.1)$ \\
& Tertiary & $7(38.9)$ \\
& Primary & $1(5.6)$ \\
Father's occupation & Secondary & $9(50.0)$ \\
& Tertiary & $8(44.4)$ \\
& Professional & $5(27.8)$ \\
& Laborer & $9(50.0)$ \\
Mother's occupation & Others & $4(22.2)$ \\
& Professional & $8(44.4)$ \\
& Not working & $10(55.6)$ \\
\hline
\end{tabular}

on researcher-developed tool assessment form. Experts were asked to respond on three components of the GIR audio including script, narration, and recording quality.

1) Script

All experts (100\%) agreed that the script was appropriate for children, comprehensible, relaxation induced and have acceptable sequencing and length.

2) Narrator

The tone of voice of the narrator, fluency and clarity was rated as acceptable by all experts. However, one expert commented on the pacing between each sentences as slow. Improvement has been made following the comment.

3) Recording quality

All experts (100\%) agreed that the recording quality and background music are good and blends appropriately.

One expert was not familiar to use MP3 player, thus a brief written guideline was developed to facilitate the users. Another expert gave suggestion that more bright and cheerful elements could be added to the script to make it sounds livelier. Based on the recommendations, necessary changes were made. Overall comments were good and the experts agreed to recommend its usage to children.

\subsection{Evaluation of Guided Imagery and Relaxation Audio}

Evaluation forms were completed by child experts' and children participants after listening to the GIR audio. The experts and participants evaluation are presented in Tables 2 and 3.

As for the experts' evaluation, all of them completed the whole audio hearing, without falling asleep and claimed to be able to have clear imagination. They also felt relaxed after hearing the audio. None of them was disturbed during the hearing session.

In addition, all children participants (100\%) completed the audio hearing. Majority of them (66.7\% - 88.9\%) was able to imagine well without falling asleep and in some way was positively affected by the imagery-induced relaxation audio. Most of them (66.7\%) claimed they were not disturbed during the hearing sessions (see Table 2).

For the overall assessment, all experts (100\%) agreed (i.e. responded Yes) to all stem of items inquired regarding the GIR audio. Likewise, majority of the children (66.7\% - 100\%) responded the same.

Almost all of the listeners agreed that the script was well written with clear description of different imaginary stimuli, and the background music was good. They admitted that they could follow through the scripts smoo-

Table 2. Percentage of experts and participants evaluation on first experience of GIR audio.

\begin{tabular}{lcccc}
\hline & \multicolumn{2}{c}{ Experts } & \multicolumn{2}{c}{$\begin{array}{c}\text { Children } \\
\text { Participants }\end{array}$} \\
\hline & Yes (\%) & No (\%) & Yes (\%) & No (\%) \\
Finished the hearing session & 100 & 0 & 100 & 0 \\
Did not fall asleep & 100 & 0 & 88.9 & 11.1 \\
Able to imagine well & 100 & 0 & 66.7 & 33.3 \\
Disturbed during the session & 0 & 100 & 33.3 & 66.7 \\
Felt affected by the & 100 & 0 & 83.3 & 16.7 \\
imagery-induced relaxation & & & & \\
\hline
\end{tabular}

Table 3. Percentage of experts and participants assessment of the GIR audio.

\begin{tabular}{lcccc}
\hline & \multicolumn{2}{c}{ Experts } & \multicolumn{3}{c}{$\begin{array}{c}\text { Children } \\
\text { Participants }\end{array}$} \\
\hline & $\begin{array}{c}\text { Not true or } \\
\text { some true (\%) }\end{array}$ & True (\%) & $\begin{array}{c}\text { Not true or } \\
\text { some true (\%) }\end{array}$ & True (\%) \\
Beneficial & 0 & 100 & 33.3 & 66.7 \\
$\begin{array}{l}\text { Good music } \\
\text { Well written script }\end{array}$ & 0 & 100 & 27.8 & 72.2 \\
$\begin{array}{l}\text { Will hear it again } \\
\text { Will recommend to }\end{array}$ & 0 & 100 & 16.7 & 83.3 \\
$\begin{array}{l}\text { friends and family } \\
\text { Able to imagine }\end{array}$ & 0 & 100 & 16.7 & 83.3 \\
$\begin{array}{l}\text { Well } \\
\begin{array}{l}\text { Able to follow the } \\
\text { flow of thoughts }\end{array}\end{array}$ & 0 & 100 & 22.2 & 77.8 \\
$\begin{array}{l}\text { Description is very } \\
\text { clear as if it is real }\end{array}$ & 0 & 100 & 0 & 100 \\
$\begin{array}{l}\text { Felt relaxed } \\
\begin{array}{l}\text { Able to focus till } \\
\text { the end }\end{array}\end{array}$ & 0 & 100 & 33.3 & 66.7 \\
\begin{tabular}{l} 
Enjoyed \\
\hline
\end{tabular} & 0 & 100 & 22.3 & 77.7 \\
\hline
\end{tabular}


thly, imagine clearly, and keep focus throughout the hearing session. As the audio was perceived as relaxing, enjoyable, and beneficial, they were happy to hear it again (see Table 3 ).

\section{Discussion}

This study consists of two phases where the aim of Phase I was to develop and evaluate a GIR audio in Bahasa Malaysia and in Phase II was to explore its effect on children with cancer. We report the research findings from Phase I only.

The first phase has been conducted in multiple stages with a lot of reconciliation and adjustment made before the final model of GIR audio was produced. As far as we are aware, the attempt to develop an imagery induced relaxation tool to be used in our pediatric population was the first of its kind. Furthermore, the script which was constructed in Bahasa Malaysia has never been produced before.

Undeniably, there might be other centers that have been using guided imagery relaxation technique that involves interaction session with therapist. However, this study develops a GIR audio in recorded format so that it is readily available to be used at anytime, even without the presence of a therapist. The tool that we have developed can either be used with MP3 players or constructed into a compact disc, whichever way makes it versatile and handy. As it does not require individual session with the therapist, it is more flexible as it can be practiced at home or even everywhere at the convenience of the patient. There is no need to set up hospital or clinic appointment with the therapist, no limited time schedule and basically the training can be carried out simultaneously for a larger number of patients at one time. Hence, it is more cost-effective as well.

In addition to that, comments and evaluations given by appointed child experts as well as the participants of this study towards the GIR audio were favorable and encouraging. This might just be a starting point towards establishing the GIR audio and many more of its kind in the future. It was an accomplishment to have developed a psychological intervention tool which is fun and enjoyable by our pediatric patients in hope to encourage and incorporate the use of psychological intervention tools in the management of our patients in the future.

\section{Conclusion and Implication}

In summary, this study has successfully become a pioneer in developing an imagery induced relaxation audio in Bahasa Malaysia which is feasible and enjoyable by children. This is the cornerstone for future incorporation of psychological therapy into the management of patients in the clinical setting. The GIR audio in Bahasa Malaysia is now ready and available to be tested on children so that the effectiveness of the tool in improving general psychological functioning can be examined. In summary, this is the first known study developing GIR audio in Bahasa Malaysia which is feasible, enjoyable and beneficial for children that warrants efficacy study.

\section{Study Limitations and Recommendations}

The findings of the current study need to be set against its limitations. The guided-imagery and relaxation script was developed and later reconstructed by only four child experts including clinical psychologist, pediatric oncologists and child psychiatrist, which may suggest a prohibitively small sample size. Nevertheless, previous work using similar techniques such as mental practice as a training strategy for laparoscopic surgery, cognitive task analysis and decision mapping also used small samples of experts [16,17]. Furthermore, extensive review of existing imagery and relaxation scripts for children has been added to the breadth of the present script. A further limitation could be the subjective evaluation obtained from both child experts and children. Whilst majority reported favorable responses upon hearing the relaxation audio, the effects of listening to the audio especially on children's state of relaxation need to be objectively measured. The second phase of the study will pilot this relaxation audio on children with cancer and examine its effectiveness. In addition, further work should consider expanding the use of guided-imagery relaxation audio in improving children's psychological well-being in different settings, such as entering first days of schooling, and facing examination. Finally, given that existing evidence indicates imagery exercises can improve self-confidence, anxiety and level of stress [18], and empirical investigation using this newly developed guided-imagery relaxation audio in Bahasa Malaysia is warranted.

\section{Acknowledgements}

The project was funded by Universiti Sains Malaysia under Short Term Grant PPSP 316/61310038.

\section{REFERENCES}

[1] M. Sawyer, G. Antoniou, I. Toogood, M. Rice and P. Baghurst, "Childhood Cancer: A 4-Year Prospective Study of the Psychological Adjustment of Children and Parents,” Journal of Pediatric Hematology/Oncology, Vol. 22, No. 3, 2000, pp. 214-220. doi:10.1097/00043426-200005000-00006

[2] E. R. Katz and S. M. Jay, "Psychological Aspects of Cancer in Children, Adolescents, and Their Families," Clinical Psychology Review, Vol. 4, No. 5, 1984, pp. 525542. doi:10.1016/0272-7358(84)90042-4

[3] J. Kellerman, “Comprehensive Psychosocial Care of the 
Child with Cancer,” In: J. Kellerman, Ed., Psychological Aspects of Childhood Cancer, C.C. Thomas, Springfield, 1980.

[4] R. Sloman, "Relaxation and Imagery for Anxiety and Depression Control in Community Patients with Advanced Cancer," Cancer Nursing, Vol. 25, No. 6, 2002, pp. 432-435. doi:10.1097/00002820-200212000-00005

[5] M. E. Broome, P. P. Lillis, T. W. McGahee and T. Bates, "The Use of Distraction and Imagery with Children during Painful Procedures," European Journal of Cancer Care, Vol. 3, No. 1, 1994, pp. 26-30. doi:10.1111/j.1365-2354.1994.tb00006.x

[6] J. A. Ellis and N. P. Spanos, "Cognitive-Behavioral Interventions for Children's Distress during Bone Marrow Aspirations and Lumbar Punctures: A Critical Review," Journal of Pain and Symptom Management, Vol. 9, No. 2, 1994, pp. 96-108. doi:10.1016/0885-3924(94)90162-7

[7] E. R. Katz, J. Kellerman and L. Ellenberg, "Hypnosis in the Reduction of Acute Pain and Distress in Children with Cancer,” Journal of Pediatric Psychology, Vol. 12, No. 3, 1987, pp. 379-394. doi:10.1093/jpepsy/12.3.379

[8] M. M. Huth, D. M. Van Kuiken and M. E. Broome, "Playing in the Park: What School-Age Children Tell Us about Imagery,” Journal of Pediatric Nursing, Vol. 21, No. 2, 2006, pp. 115-125. doi:10.1016/j.pedn.2005.06.010

[9] C. L. Baird, M. M. Murawski and J. Wu, "Efficacy of Guided Imagery with Relaxation for Osteoarthritis Symptoms and Medication Intake,” Pain Management Nursing, Vol. 11, No. 1, 2004, pp. 56-65. doi:10.1016/j.pmn.2009.04.002

[10] M. H. Hockenberry, "Guided Imagery as a Coping Measure for Children with Cancer," Journal of Pediatric Oncology Nursing, Vol. 6, No. 2, 1989, p. 29. doi:10.1177/104345428900600219

[11] C. Liossi and P. Hatira, "Clinical Hypnosis versus Cognitive Behavioral Training for Pain Management with Pediatric Cancer Patients Undergoing Bone Marrow Aspira- tions,” International Journal of Clinical and Experimental Hypnosis, Vol. 47, No. 2, 1999, pp. 104-116. doi:10.1080/00207149908410025

[12] S. Manne and M. Andrykowski, “Are Psychological Interventions Effective and Accepted by Cancer Patients? II. Using Empirically Supported Therapy Guidelines to Decide,” Annals of Behavioral Medicine, Vol. 32, No. 2, 2006, pp. 98-103. doi:10.1207/s15324796abm3202 4

[13] R. W. Olmsted, L. Zeltzer and S. LeBaron, "Hypnosis and Nonhypnotic Techniques for Reduction of Pain and Anxiety during Painful Procedures in Children and Adolescents with Cancer," The Journal of Pediatrics, Vol. 101, No. 6, 1982, pp. 1032-1035. doi:10.1016/S0022-3476(82)80040-1

[14] K. Schmidt and E. Ernst, "Assessing Websites on Complementary and Alternative Medicine for Cancer," Annals of Oncology, Vol. 15, No. 5, 2004, pp. 733-742. doi:10.1093/annonc/mdh174

[15] J. T. Lusk, "30 Scripts for Relaxation, Imagery and Inner Healing, Vol. 1,” Whole Person Associates, Minnesota, 1992.

[16] K. A. Ericsson, "Protocol Analysis and Expert Thought: Concurrent Verbalization of Thinking during Experts' Performance on Representative Thoughts,” In: K. A. Ericsson, N. Charness, P. J. Feltovich and R. R. Hoffman, Eds., The Cambridge Handbook of Expertise and Expert Performance, Cambridge University Press, Cambridge, 2006. doi:10.1017/CBO9780511816796.013

[17] S. Arora, R. Aggarwal, N. Sevdalis, A. Moran, P. Sirimanna, R. Kneebone and A. Darzi, "Development and Validation of Mental Practice as a Training Strategy for Laparoscopic Surgery,” Surgical Endoscopy, Vol. 24, No. 1, 2010, pp. 179-187. doi:10.1007/s00464-009-0624-y

[18] R, Weinberg, "Does Imagery Work? Effects on Performance and Mental Skills,” Journal of Imagery Research in Sport and Physical Activity, Vol. 3, No. 1, 2008, pp. 1-21. doi:10.2202/1932-0191.1025 